\title{
Sustitución de tecnologías en Máquinas Herramientas: caso de la industria litográfica en semiconductores 1962-2010
}

\author{
Substitution of technologies in Machine Tools: case of lithographic industry in semicodutors \\ $1962-2010$
}

\author{
Luis José Llaque Ramos ${ }^{1}$
}

\begin{abstract}
Resumen
El objetivo de este estudio fue establecer cuáles son los factores que permiten explicar la longevidad de las tecnologías en la industria litográfica de máquinas herramientas de semiconductores bajo el enfoque de Murmann- Frenken y la teoría de la complejidad. El método utilizado se basa en un modelo que permite el análisis de los componentes y los complementos de estas tecnologías a lo largo de seis generaciones desde 1962 hasta el 2010. Los resultados muestran que la larga vida de las tecnologías de la industria de máquinas herramientas en litografía se explica por la existencia de mejoras en componentes periféricos (como las herramientas de grabado y/o piezas ópticas), que ha permanecido en actividad por más de 20 años en cinco de las seis generaciones tecnológicas analizadas. Asimismo, se encuentra que la elevada velocidad de evolución de los complementos secundarios (como máscaras) es otro factor que explica la sustitución de tecnologías, algo que también sucede en otras industrias de productos complejos.
\end{abstract}

Palabras clave: complementos; componentes; máquinas herramientas; evolución tecnológica; complementos.

\begin{abstract}
The objective of this study is to establish the factors that explain the longevity of technologies in the lithographic industry of semiconductor machine tools under the Murmann-Frenken approach and the theory of complexity. The method used for this longitudinal study is based on a model that allows the analysis of the components and complements of these technologies over six generations from 1962 to 2010. The results show that the long life of the technologies of the machine tool industry in lithography is explained by the existence of improvements in peripheral components (such as engraving tools and / or optical parts), which has been active for more than 20 years in five of the six technological generations analyzed. Also, it is found that the high speed of evolution of secondary complements (such as masks) is another factor that explains the substitution of technologies, something that also happens in other industries of complex products.
\end{abstract}

Keywords: hierarchy of components; machine tools; technological evolution; complement.

\section{Introducción}

La sustitución de tecnologías complejas es parte del progreso en una economía. Esta sustitución ha sido conceptualizada en la literatura de la tecnología, a través de modelos cíclicos, en el cual la tecnología evoluciona desde un período de introducción y experimentación; o un período de fermento, donde se presenta una cantidad significativa de incertidumbre tecnológica y de mercado; luego se pasa a una etapa de crecimiento, donde emerge un diseño dominante, el cual reduce de manera importante la incertidumbre tecnológica que anuncia el comienzo de la etapa de cambio incremental en los componentes de menor jerarquía (Tushman y Anderson,1990; Tushman y Rosenkopf, 1992; Murmann and Frenken, 2006). Cuando el componente de mayor jerarquía no es capaz de evolucionar, surge entonces una nueva tecnología que sustituye a la anterior con un mayor rendimiento.

La importancia de comprender la sustitución de tecnologías complejas, como las máquinas herramientas de litografía, desde los años ochenta hasta los primeros 12 años del siglo XXI, es que estas han brindado soluciones, en más del 90\% en la industria de semiconductores (Schwab, 2017). Asimismo, el sector de máquinas herramientas, resulta importante y es capital en cualquier economía, ya que históricamente se ha demostrado que es el punto de partida del aprendizaje y difusión de la tecnología (Rosenberg, 1979).

El objetivo del trabajo es establecer cuales son los factores que permiten explicar la longevidad de las tecnologías en la industria litográfica de máquinas herramientas de semiconductores bajo el enfoque histórico y de la teoría de la complejidad.

\section{Materiales y métodos}

El desarrollo de este trabajo se basó en la construcción de un modelo para el análisis de las sustituciones tecnológicas, el cual recoge los fundamentos teóricos de Murmann and Frenken (2006) y Adner and Kapoor (2016). 
Tabla 1. Modelo de análisis para la sustitución de máquinas herramientas en litografía en semiconductores

\begin{tabular}{|c|c|}
\hline $\begin{array}{c}\text { Componentes Principales (Fuente } \\
\text { de Energía, hornos de difusión) } \\
\text { b1. }<0\end{array}$ & $\begin{array}{c}\text { Componentes Periféricos o } \\
\text { secundarios (Piezas ópticas } \\
\text { y/o herramientas de grabado) } \\
\text { b1. }<0\end{array}$ \\
\hline $\begin{array}{c}\text { Complementos Principales } \\
\text { (Resist) } \\
\text { b2. }<0\end{array}$ & $\begin{array}{c}\text { Complementos secundarios } \\
\text { (Máscaras) } \\
\text { b2 }>0\end{array}$ \\
\hline
\end{tabular}

Del modelo anteriormente propuesto en la Tabla 1, se desprenden las siguientes relaciones: Componentes= componentes principales + componentes secundarios. Complementos $=$ Complementos principales + complementos secundarios.

\section{Hipótesis}

Sustitución tecnológica $=a+$ b1.Componentes + b2.complementos

1) Si $\mathbf{b}_{\mathbf{1}}<\mathbf{0}$, implica que los cambios de componentes de mayor jerarquía (fuentes de energía) reducen el tiempo de vida de la tecnología de máquinas herramientas, conduciendo a una baja participación de mercado, para el fabricante. 2) $\mathbf{S i} \mathbf{b}_{\mathbf{1}}>\mathbf{0}$, implica que los cambios de componentes de menor jerarquía (piezas ópticas y/o herramientas de grabado) aumentan el tiempo de vida de la tecnología de máquinas herramientas, conduciendo a mantener una mayor participación de mercado, para el fabricante. 3) Si $\mathbf{b}_{2^{\bullet}}<\mathbf{0}$, significa que los cambios en los complementos principales (resist) acortan el tiempo de vida de la tecnología; esto debe ser entendido como que la sustitución de complementos principales resulta ser muy costosa en relación a la nueva tecnología que muestra igual o un mayor rendimiento, conduciendo a una menor participación de mercado. 4) Si $\mathbf{b}_{2}>\mathbf{0}$, significa que los cambios en los complementos secundarios (máscaras), permiten alargar el tiempo de vida de la tecnología; esto significa, que la participación de mercado de las máquinas herramientas, se incrementa, porque nuevas máscaras (de bajo precio, en relación a la resist) permiten elaborar nuevos tipos de chips. 5) Si a. $<\mathbf{0}$, significa que los compradores de máquinas herramientas tienen expectativas negativas (con respecto a la tecnología actual), es decir no hay una inercia, debido a que consideran que la demanda del mercado exige tecnologías cualitativamente más avanzadas, por lo cual consideran que deben invertir en nuevas tecnologías, por temor a la pérdida de competitividad. 6) $\mathbf{S i} \mathbf{a}>\mathbf{0}$, significa que los compradores de máquinas herramientas tienen expectativas positivas (con respecto a la tecnología actual), es decir hay una inercia, debido a que en los fabricantes de máquinas herramientas han surgido varias mejoras de la tecnología actual, por lo cual consideran que no deben invertir en nuevas tecnologías, ya que con el cambio de componentes periféricos y/o complementos, pueden alargar la vida de la tecnología actual.

Data: El proceso que se siguió para los datos de las regresiones consistió en considerar la evolución de la eficiencia de los componentes (a partir del número de transistores por chip, con respecto a la generación tecnológica anterior), este procedimiento se siguió para las tecnologías Contac Printer (de 1970, medida con respecto a los niveles de 1962), la tecnología Proximity Printer (de 1970, con respecto a los niveles de la tecnología Contac Printer de 1970); la tecnología Scanning Projection (de 1972, medida con respecto a los niveles de la tecnología Proximity Printer de 1972). Este procedimiento ha sido seguido anteriormente por Balcer y Lippman (1984) y Farzin et al. (1998).

Para los complementos de estas tres tecnologías, se midió a partir del cociente entre los gastos en máscaras de impresión y el costo de cada máquina herramienta; esto nos proporcionó el indicador de la innovación o mejora de la eficiencia de los complementos. La variable dependiente, en todos los casos se consideró la participación de mercado de la respectiva tecnología de máquinas herramientas.

Para las tecnologías G- line Stepper; I-line Stepper y DUV-248Stepper, la eficiencia de los componentes se construyó a partir del área de imagen de grabado sobre la oblea o chip. En la tecnología G- line Stepper, se midió con respecto al área de grabado de 1976. Para la tecnología Iline Stepper, se midió con respecto al área de tecnología Gline Stepper de 1985. En la tecnología DUV-248 Stepper, se midió con respecto al área de la tecnología I-line Stepper de 1986.

En la construcción de los indicadores de mejoras en los complementos para estas tres tecnologías, se siguió el mismo procedimiento señalado anteriormente. Las tecnologías E-beamWriter(1976) y X-ray Printer (1978), no encontraron demanda en el mercado, dado que requerían de nuevas fuentes de energía y nuevos complementos; ambas tecnologías no requerían de piezas ópticas. La tecnología DUV-193 Stepper (1996) y DUV 193- Inmersion (2005), no presentan datos disponibles a nivel cuantitativo.

La base de datos utilizada procede de Adner and Kapoor (2010), VLSI Research (1992), Pieczulewski (1995), Nikon (2005), Kim (2014) y Marcher \& Mowery (2006).

\section{Resultados y discusión}

La Litografía de Maquinas Herramientas en $\underline{\text { Semiconductores }}$

La litografía de semiconductores es el proceso por el que un diseño de un circuito se imprime en un sustrato de un semiconductor llamado oblea (o wafer). Después del diseño de un circuito integrado (CI) se finaliza (es decir el cableado, las puertas y las uniones), el modelo de circuito es transferirlo a una "máscara" (Adner and Kapoor,2010; Henderson, 1995).

En la fabricación de máquinas herramientas de la industria de la litografía (que fabrica Chips) los componentes principales de estas máquinas son las fuentes de energía y los sistemas ópticos y/o herramientas de grabado son los componentes periféricos; en tanto que los complementos secundarios son los dispositivos denominados máscaras y los complementos principales son la resist (conformado por productos químicos). 


\section{Estimaciones}

La Tabla 2 proporciona los resultados de los factores que afectan la longevidad de la tecnología de máquinas herramientas, tanto para la tecnología Contac Printer, Tecnología Proximity Printer y la Tecnología Scanning Projection.

La estimación de la columna uno muestra que el período de vida de la tecnología Contac Printer (19701987) depende de la innovación de los componentes (coeficiente 2,7887) en una mayor proporción en relación a los complementos (coeficiente 0,963 ). Los signos positivos obtenidos, nos permiten verificar nuestras hipótesis (dos y cuatro) en nuestro modelo, en la cual la innovación de componentes y complementos impulsan la participación de mercado de estas tecnologías. Asimismo, se aprecia que los componentes (como herramientas de grabado) tienen un mayor impacto en la longevidad de tecnología, en relación a los complementos periféricos (como máscaras). El término independiente, no resulta significativo, desde el punto de vista estadístico.

Tabla 2. Factores que afectan la longevidad en máquinas herramientas en Litografía

\begin{tabular}{lccc}
\hline $\begin{array}{l}\text { Regresión } \\
\text { OLS }\end{array}$ & $\begin{array}{c}\text { Tecnología } \\
\text { Contac Printer } \\
(1)\end{array}$ & $\begin{array}{c}\text { Tecnología } \\
\text { Proximity } \\
\text { Printer } \\
(2)\end{array}$ & $\begin{array}{c}\text { Tecnología } \\
\text { Scanning } \\
\text { Projection } \\
(3)\end{array}$ \\
\hline Constante & 1,514 & $-0,707$ & $-25,575$ \\
$\mathrm{t}$ & $(0,211)$ & $(-0,301)$ & $(-4,286)$ \\
Componentes & 2,7887 & $-0,48$ & 1,038 \\
$\mathrm{t}$ & $(2,407)$ & $(-1,327)^{*}$ & $(1,016)^{*}$ \\
Complementos & 0,963 & 1,701 & 3,361 \\
$\mathrm{t}$ & $(2,193)$ & $(4,82)$ & $(7,807)$ \\
R cuadrado & 0,525 & 0,638 & 0,885 \\
$\mathrm{~F}$ & 8,29 & 14,971 & 61,423 \\
Observaciones & 18 & 20 & 19 \\
\hline Fue & & &
\end{tabular}

Fuente: Regresión utilizando SPSS

Los valores en parentesis son las distribuciones $t$, los parámetros son significativos al 5\%

(*) Significativo al $15 \%$

En la columna dos de la Tabla 2, se aprecia los resultados de la evaluación del modelo propuesto, para la tecnología Proximity Printer (1970-1989), en la que se encuentra que el cambio en componentes principales (como hornos de difusión), con un coeficiente de $(-0,48)$ reducen el tiempo de vida de la tecnología, conduciendo a una baja participación de mercado, verificando la hipótesis uno. Sin embargo, las innovaciones en los complementos (máscaras), con un coeficiente de $(1,701)$ permiten a largar el tiempo de vida de la tecnología, verificando la hipótesis cuatro de nuestro modelo. El término independiente, en esta regresión no resulta significativo, desde el punto de vista estadístico.

Para la columna tres, de la Tabla 2 se muestra que el período de vida de la tecnología Scanning Proyection (1972-1990) depende de la innovación de complementos (coeficiente 3,361) en una mayor proporción que la innovación de componentes (coeficiente 1,038). Los signos positivos obtenidos, nos permiten afirmar que los componentes periféricos y los complementos secundarios son los factores que sustentan la longevidad de esta tecnología, es decir, dan sustento a las hipótesis dos y cuatro del modelo propuesto. El término independiente, en esta regresión si resulta significativo, desde el punto de vista estadístico. La lectura de este $(-25,575)$, no permite verificar la hipótesis 5, sobre la existencia expectativas negativas sobre el desempeño de la tecnología actual; por lo cual el fabricante de máquinas herramientas requiere adoptar una nueva tecnología, para seguir siendo competitivo en el mercado.

Tabla 3. Factores que afectan la longevidad en máquinas herramientas en Litografía

\begin{tabular}{|c|c|c|c|}
\hline Regresión & Tecnología & Tecnología & Tecnología \\
\hline OLS & $\begin{array}{c}\text { G - line Stepper } \\
(1978-1998) \\
(1)\end{array}$ & $\begin{array}{l}\text { I- line Stepper } \\
(1985-2010) \\
\text { (2) }\end{array}$ & $\begin{array}{c}\text { DUV } 248 \text { Stepper } \\
(1986-2010) \\
(3)\end{array}$ \\
\hline Constante & 13,874 & 4,375 & $-2,551$ \\
\hline $\mathrm{t}$ & $(3,971)$ & $(1,121)$ & $(-0,391)$ \\
\hline Componentes & 1,62 & 1,359 & 0,262 \\
\hline $\mathrm{t}$ & $(2,031)$ & $(10,938)$ & $(1,812)$ \\
\hline Complementos & 3,073 & 1,1 & 1,833 \\
\hline $\mathrm{t}$ & $(2,235)$ & $(2,378)$ & $(2,516)$ \\
\hline$R$ cuadrado & 0,789 & 0,841 & 0,619 \\
\hline $\mathrm{F}$ & 33,647 & 60,768 & 17,907 \\
\hline Observaciones & 21 & 26 & 25 \\
\hline
\end{tabular}

Fuente: Regresión utilizando SPSS

Todos los parámetros son significativos al 5\%; los valores en parentesis son las distribuciones $\mathrm{t}$

La Tabla 3 proporciona los resultados de los factores que afectan la longevidad de la tecnología (el período de vida, en el mercado) de máquinas herramientas, tanto para la tecnología G -line Stepper, Tecnología I - line Stepper y la Tecnología DUV-248 Stepper, La estimación en la columna uno (Tabla 3) muestra que el período de vida de la tecnología $G$ depende de la evolución de los complementos secundarios (coeficiente 3,073) y los componentes periféricos (coeficiente 1,62), los signos positivos obtenidos, nos permiten verificar nuestras hipótesis (verificando las hipótesis dos y cuatro, según el modelo propuesto) sobre los componentes que impulsan el período de vida en el mercado para esta tecnología. Asimismo, se aprecia que los complementos secundarios (las máscaras) tienen un mayor impacto en la longevidad de la tecnología, en relación al impacto de los componentes periféricos (las piezas ópticas). El término independiente $(13,874)$ presenta un signo positivo, lo cual nos permite verificar la hipótesis 6 del modelo propuesto.

Para la tecnología I- line, en la columna dos, se muestra, que estas máquinas herramientas dependen de la evolución de los componentes periféricos (coeficiente 1,359) en mayor proporción que la evolución de los complementos secundarios (coeficiente 1,100). Los signos positivos, de ambos coeficientes (verifican las hipótesis dos y cuatro, según nuestro modelo) alargan el período de vida de la tecnología, por un período mayor a la tecnología $\mathrm{G}$-line. 
La columna tres de la Tabla 3, muestra que la longevidad de la tecnología DUV-248 Stepper depende positivamente de los componentes periféricos (coeficiente 0,262), lo cual verifica la hipótesis dos del modelo, en relación a que un cambio en los componentes periféricos aumenta el tiempo de vida de la tecnología. El coeficiente positivo de los complementos (coeficiente 1,833) permite sustentar, según nuestro modelo, que los complementos secundarios, tienen un mayor impacto en la longevidad de la tecnología DUV248Stepper, en relación a los componentes (verificando la hipótesis cuatro).

La Tabla 4 muestra la importancia de los componentes y/o complementos (cuando se incrementa en un 10\%) en la sustitución de tecnologías para las máquinas herramientas en litografía. De las seis tecnologías mostradas, en promedio los componentes (con una innovación del 10\%) contribuyeron en un 10,9\% en el aumento de la duración de las tecnologías para el período 1970-2010. En tanto que los complementos (con una innovación del 10\%) impulsaron el aumento en el período de vida de las tecnologías en promedio en un $20 \%$.

Tabla 4. Importancia de la Innovación en Componentes y Complementos en el caso de la Sustitución de Tecnologías con una mejora del $10 \%$ en componentes y/o Complementos

\begin{tabular}{llrr}
\hline \multicolumn{1}{c}{ Tecnología } & $\begin{array}{l}\text { Permanencia } \\
\text { Comercial } \\
(* * *)\end{array}$ & Componentes & Complementos \\
& 18 años & 27,8 & 9,6 \\
1. Contac Printer & 20 años & $-4,8$ & 17,01 \\
2. Proximity Printer & & 11,5 & 13.305 \\
Promedio (1 y2)* & & 10,1 & 33,6 \\
3. Scanning Projection 19 años & 16,2 & 30,7 \\
4. G -line Stepper & 21 años & 13,5 & 11 \\
5. I-line Stepper & 26 años & 13,26 & 25,1 \\
Promedio (3, 4 y 5)* & & 2,6 & 18,3 \\
6. DUV- 248 Stepper & & 10,9 & 20,035 \\
Promedio Total ** & - & & \\
Fuente: Tabla 2 y 3. & & &
\end{tabular}

(*)Cuantifica la velocidad de innovación en componentes y complementos (**) Cuantifica la velocidad promedio de innovación en componentes y complementos de las 6 tecnologías

$(* * *)$ Tiempo aproximado de comercialización de las máquinas herramientas de litografía

Las tecnologías Contac printer y Proximity Printer, aparecieron en los años setenta, a nivel comercial; en este período, los mismos fabricantes de Chips, estaban obligados a construir sus propias máquinas herramientas, debido a que en el mercado no existían proveedores; la razón de la escasez de estos se explica, según Pillai (2017), y Marcher and Mowery (2004) por lo reducido del tamaño del mercado, que no garantizaba economías de escala. En este período, según nuestras estimaciones, un incremento del $10 \%$ en la tasa de innovación de componentes en promedio alarga el período de vida de estas tecnologías en $11,5 \%$. Asimismo, un incremento del $10 \%$ en la tasa de innovación de los complementos, extiende la vida de estas tecnologías en 13,305\% (Tabla 4). La demanda, por el lado industrial y comercial, estaba impulsado por los fabricantes de calculadoras, relojes y los fabricantes de electrodomésticos (como hornos, televisores y lavadoras).

Las tecnologías Scanning Projection, G- line Stepper e I - line Stepper, muestran que la innovación de componentes en un $10 \%$ en promedio, extienden la vida de estas tecnologías en un 13,26\%; en tanto que una innovación de $10 \%$ en los complementos, prolongan la vida de estas tecnologías en un 25,1\% (Tabla 4). El comportamiento de estas tecnologías en este período, se encuentra explicado por varias razones: 1) La mayor demanda de microprocesadores, para la fabricación de computadoras de mesa y estaciones de trabajo por las empresas a nivel general; 2) la aparición de proveedores de componentes (piezas ópticas), como Hitachi, Nikon, Canon, Zeiss; y proveedores de complementos como máscaras (empresas especializadas en elaborar software para diseño) y (productos químicos). Sin embargo, la elevada tasa de innovación en los complementos, reside según Adner and Kapoor (2016), en el bajo costo que tienen las máscaras, en relación a los componentes. Asimismo, la posibilidad de utilizar la misma fuente de energía, con las mismas máquinas herramientas ( $\mathrm{G}$ - line y I - line), por parte de los fabricantes de Chips. En el caso de la innovación de componentes, nuestros resultados han mostrado que ha sido las innovaciones en las piezas ópticas (componentes periféricos), las que han impulsado la extensión del período de vida de estas tecnologías; estos mismo resultados son señalados desde el punto de vista cualitativo por Brunner (2003); Henderson (1995), Henderson and Clark (1990) y Hutcheson (2008). Las innovaciones en las piezas ópticas, impulsaron dos versiones de las máquinas herramientas Gline stepper y tres generaciones distintas de las máquinas herramientas I- line stepper (Pillai, 2017). Un tercer factor, que impulso, el desarrollo se encuentra en los gastos de defensa de los EE.UU, el denominado programa militar, de la Guerra de las Galaxias, en la era Reagan 1981-1989 (Broad, 1986).

Para la tecnología DUV-248 Stepper, un aumento en su tasa de crecimiento en la innovación de complementos, (Tabla 4), de 10\%; permite alargar la vida de la tecnología en $18,3 \%$.

Las razones del fuerte impacto se encuentra en tres factores: el crecimiento de la demanda de microprocesadores (para estaciones de trabajo); el desarrollo de proveedores (de sistemas ópticos y de máscaras) especializados producto de la desintegración vertical de los fabricantes de semiconductores (Marcher and Mowery, 2004) y la cooperación de empresas americanas (a través de SEMATECH) para la inversión en investigación $\mathrm{y}$ desarrollo de las próximas generaciones de máquinas herramientas de fotolitografía, desde 1988 (Pillai, 2013). El bajo impacto de las tasa de innovación en componentes $(2,6 \%)$ en la prolongación del ciclo de vida de esta tecnología se explica, en primer lugar por el elevado costos de los componentes y las nuevas generaciones tecnológicas de equipos de litografía (Anexo 1). En segundo lugar, las foundries (empresas que fabrican Chips, por contrato solamente), redujeron el costo de producción, como consecuencia de tener acceso a licencias de fabricación 
de empresas fabless (empresas especializadas en diseñar y comercializar chips) principalmente; y finalmente el desarrollo de importantes mejoras en los software de diseño para la simulación de nuevos productos con semiconductores, que han incrementado las capacidades de simulación por computadoras disponibles. Asimismo, las herramientas de diseño electrónico y bibliotecas de células también apoyan el diseño de chips más complejos (Marcher y Mowery, 2004).

La Tabla 5 nos permite establecer los aportes de otras investigaciones sobre sistemas complejos, y contrastarlos con los resultados obtenidos. Desde Henderson y Clark (1990), la mejora de la industria de litografía en máquinas herramientas se ha encontrado impulsada por las innovaciones de componentes periféricos; este trabajo no señala, en ningún momento la importancia de los complementos; su análisis está basado en entrevistas, su fuente es fundamentalmente cualitativa. Para Henderson (1995), la evolución de la litografía para el período 1970-1990; se encuentra que tanto las innovaciones en componentes periféricos (piezas ópticas), como los cambios en los complementos (principales y secundarios) son los que explican la larga duración de la litografía de máquinas herramientas en la industria de semiconductores.

Adner y Kapoor (2016), señalan que los componentes periféricos permitió extender el uso de la tecnología Gline y retrasar el uso de la siguiente generación tecnológica (I- line). Por el lado de los complementos, estos investigadores presentan como explicación de la larga vida de las tecnologías G- line Stepper e I-line Stepper la intercambiabilidad de los complementos principales y secundarios.

Por otro lado, Henfridsson et al. (2009), muestran que en la industria automotriz la innovación de las empresas se encuentra orientada por el lado de los complementos (tipo de software que utiliza cada marca), ya que la innovación de componentes es muy cercana entre empresas. Así, señalan que una empresa automotriz, con un auto, con la misma cantidad de componentes (aproximadamente) se diferencia de las demás, porque los complementos (el software que utiliza) diferenciados entregan más beneficios a los usuarios del producto. En esa misma dirección, la tecnología I- line, ha permanecido un mayor tiempo en el mercado, dado que las máscaras (complementos secundarios), que se utilizaban en la tecnología G- line, también se podían utilizar para fabricar Chips.

Desde el punto de vista económico, la sustitución de tecnologías en máquinas herramientas, guarda coherencia con la búsqueda de una mayor rentabilidad, y no la de eficiencia tecnológica, estos se explica porque tanto la tecnología I- line, como la tecnología DUV-248Stepper, permanecieron congeladas por más de siete años, para lograr una hegemonía de mercado que según nuestras estimaciones (Figura 1, 2 y 3), solo duran alrededor de siete años; hasta que la demanda de mercado de nuevos tipos de chips, se vuelve más exigente, y se da la sustitución. Sin embargo, es importante señalar, que en el mercado de semiconductores, en la fase de transición, o en la etapa de diseño dominante por una tecnología, las tecnologías antiguas continúan produciendo chips, que atienden a

nichos de mercado. Dado que los propietarios de máquinas herramientas no las desechan, sino que continúan trabajando, aun cuando se encuentran totalmente depreciadas.

Tabla 5. Importancia de los complementos y componentes en Sistema Complejos

\begin{tabular}{|c|c|c|c|c|c|c|}
\hline Autores & $\begin{array}{c}\text { Henderson y Clark } \\
(1990)\end{array}$ & $\begin{array}{l}\text { Henderson } \\
\text { (1995) }\end{array}$ & $\begin{array}{l}\text { Funk } \\
(2009)\end{array}$ & $\begin{array}{c}\text { Adner y Kapoor } \\
(2016)\end{array}$ & $\begin{array}{l}\text { Llaque } \\
(2018)\end{array}$ & $\begin{array}{l}\text { Henfridsson et al. } \\
(2009)\end{array}$ \\
\hline Componentes & $\begin{array}{l}\text { La mejora en los } \\
\text { componentes ha sido } \\
\text { factor que ha impulsado } \\
\text { las innovaciones en la } \\
\text { industria de máquinas } \\
\text { herramientas de litografía, } \\
\text { específicamente los } \\
\text { componentes periféricos } \\
\text { (piezas ópticas), lo cual } \\
\text { ha significado que las } \\
\text { máquinas que aceptan } \\
\text { nuevos componentes, } \\
\text { incrementan su ciclo de } \\
\text { vida }\end{array}$ & $\begin{array}{l}\text { Identifica que los } \\
\text { componentes (los } \\
\text { dispositivos ópticos) } \\
\text { son los que han } \\
\text { mejorado en las } \\
\text { máquinas, y permiten } \\
\text { extender el periodo } \\
\text { de vida, su análisis es } \\
\text { para el periodo 1970- } \\
1990\end{array}$ & $\begin{array}{l}\text { Encuentra que las } \\
\text { mejoras incrementales } \\
\text { de los componentes } \\
\text { de las cintas de audio } \\
\text { y video han creado } \\
\text { discontinuidades } \\
\text { tecnológicas tanto } \\
\text { en las cintas como } \\
\text { en los equipos de } \\
\text { reproducción }\end{array}$ & $\begin{array}{l}\text { Las mejoras de } \\
\text { los componentes } \\
\text { periféricos } \\
\text { (piezas ópticas) } \\
\text { permitió extender } \\
\text { el uso de la G } \\
\text {-line con relación } \\
\text { a la tecnología } \\
\text { I- line }\end{array}$ & $\begin{array}{c}\text { Encuentra que las } \\
6 \text { generaciones } \\
\text { tecnológicas } \\
\text { analizadas desde } \\
1970 \text { hasta } 2010, \\
\text { la innovación de } \\
\text { los componentes } \\
\text { periféricos es uno } \\
\text { de los factores que } \\
\text { explica la longevidad } \\
\text { de la tecnología }\end{array}$ & $\begin{array}{l}\text { La mejora de los } \\
\text { componentes es } \\
\text { parcialmente uniforme en } \\
\text { todas las grandes empresas } \\
\text { automotrices, ya que estas } \\
\text { mayormente se encuentran } \\
\text { producidas por los mismos } \\
\text { proveedores. }\end{array}$ \\
\hline Complementos & $\begin{array}{l}\text { No realiza ningún análisis } \\
\text { específico sobre los } \\
\text { complementos }\end{array}$ & $\begin{array}{l}\text { Considera tanto a } \\
\text { los complementos } \\
\text { principales( la } \\
\text { resist), como a } \\
\text { los complementos } \\
\text { periféricos (máscaras) } \\
\text { importantes en } \\
\text { la duración de la } \\
\text { tecnología litográfica } \\
\text { para el periodo 1970- } \\
\text { 1990, desde un punto } \\
\text { de vista cualitativo }\end{array}$ & $\begin{array}{l}\text { No realiza análisis } \\
\text { sobre complementos }\end{array}$ & $\begin{array}{c}\text { Encuentran } \\
\text { que los mismos } \\
\text { complementos } \\
\text { principales } \\
\text { y periféricos } \\
\text { continuaron } \\
\text { utilizándose tanto } \\
\text { en la tecnología } \\
\text { G-line como en } \\
\text { la tecnología } \\
\text { I- line. }\end{array}$ & $\begin{array}{l}\text { Encuentra que de } \\
\text { las } 6 \text { generaciones } \\
\text { analizadas, la } \\
\text { velocidad de } \\
\text { innovación de los } \\
\text { complementos es el } \\
\text { doble que la de los } \\
\text { componentes }\end{array}$ & $\begin{array}{l}\text { La ventaja competitiva } \\
\text { de las empresas en la } \\
\text { industria automotriz, } \\
\text { se encuentran por el } \\
\text { lado de la integración } \\
\text { de componentes que } \\
\text { aceptan componentes } \\
\text { funciones distintas y } \\
\text { diferen ciadas. Esta } \\
\text { diferenciación se logra } \\
\text { a través de dotar a los } \\
\text { componentes con nuevos } \\
\text { complementos (diferentes } \\
\text { tipos de software) a los } \\
\text { automóviles. }\end{array}$ \\
\hline
\end{tabular}




\section{Conclusiones}

El modelo propuesto ha permitido probar que la sustitución de tecnologías en máquinas herramientas para litografía se encuentra explicada por las innovaciones en los complementos (en una mayor proporción) en relación a los componentes. Así, las innovaciones en complementos periféricos (máscaras) han mostrado una mayor tasa de innovación, sustentada en su bajo costo, y en el crecimiento de proveedores especializados. El modelo propuesto también ha permitido estimar de manera específica que la prolongación del ciclo de vida de las tecnologías G-line, I-line y DUV-248, se encuentran explicados tanto por las innovaciones en los complementos como por los componentes periféricos; y que la velocidad de innovación de los complementos es en promedio el doble que la velocidad de innovación de los componentes (para las 6 tecnologías analizadas).

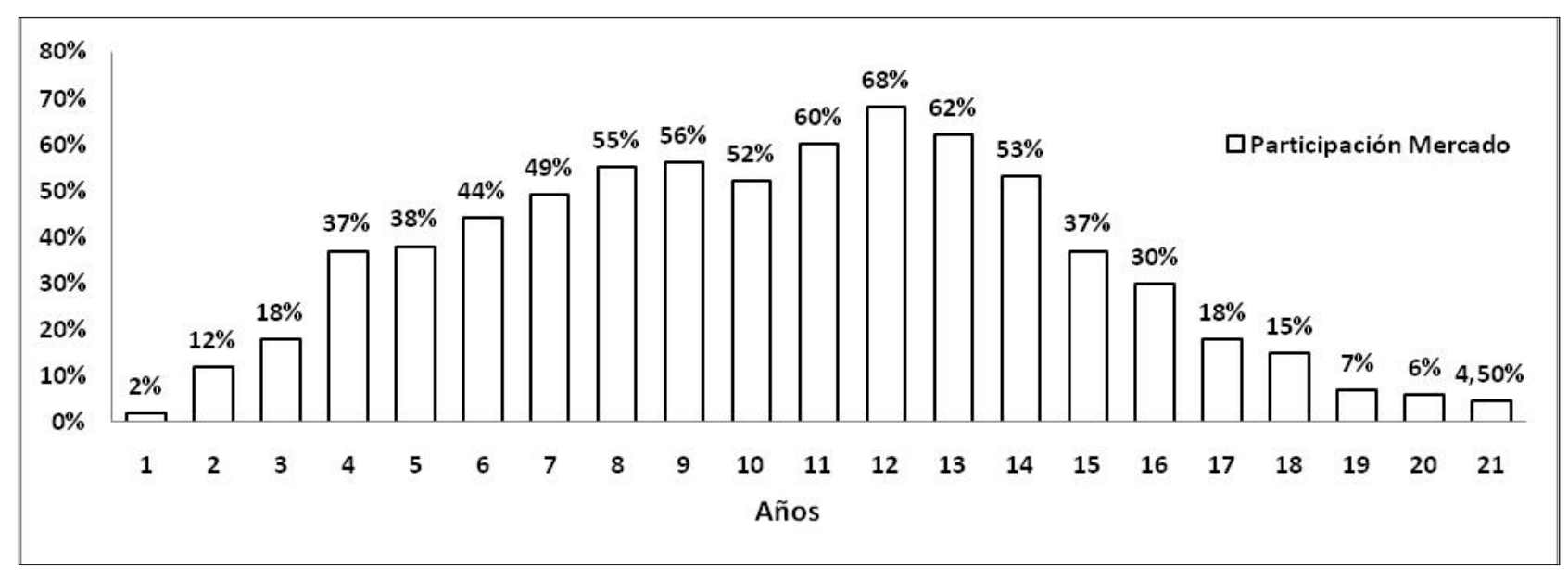

Figuera 1. Partición de mercado de la tecnología en maquinas herramientas G - line Stepper

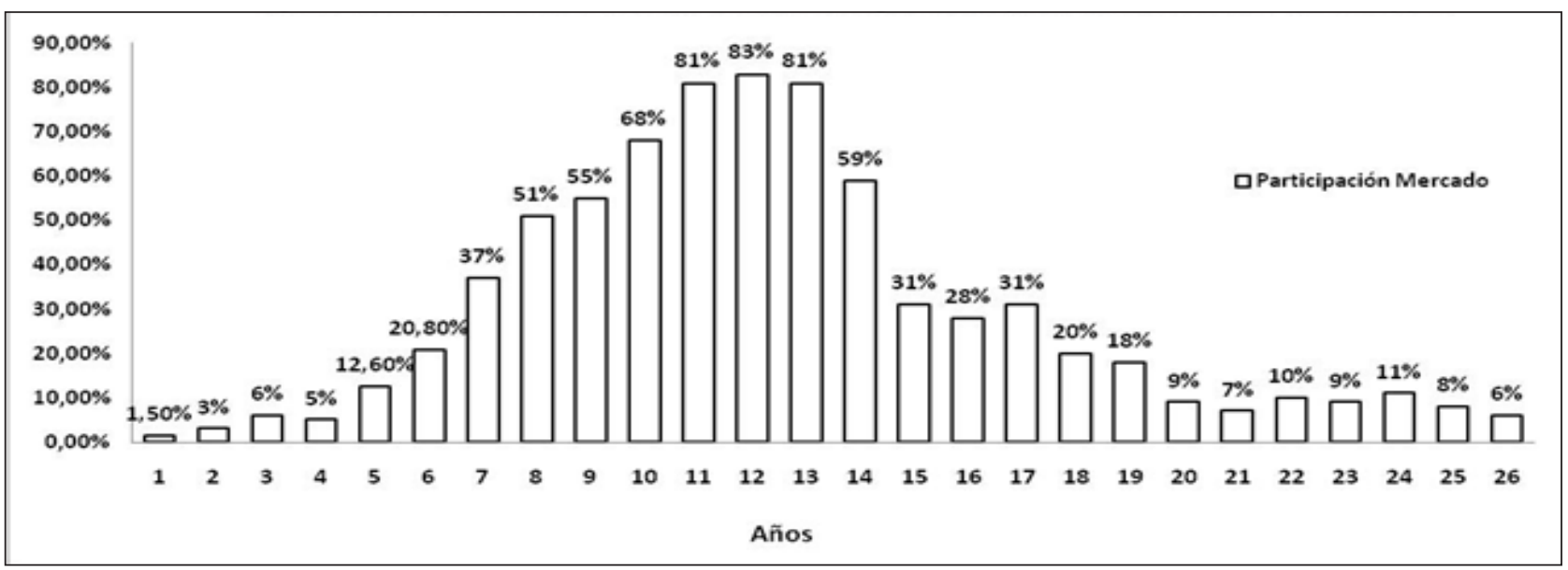

Figuera 2. Partición de mercado de la tecnología en maquinas herramientas I - line Stepper

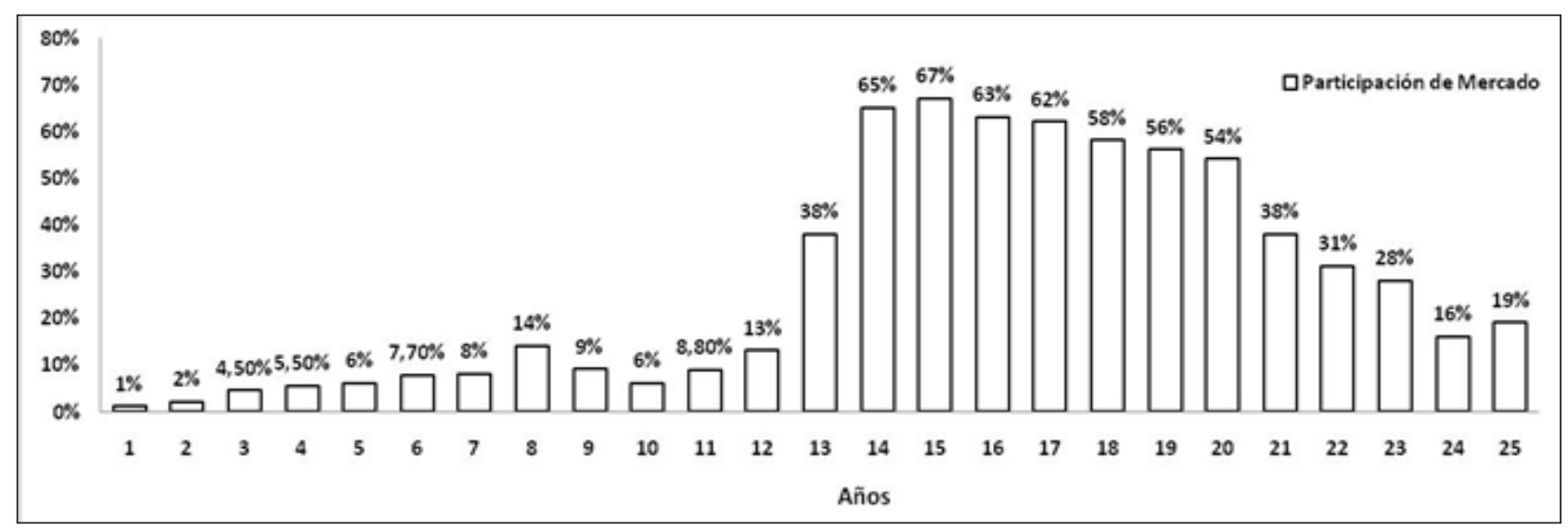

Figuera 3. Partición de mercado de la tecnología en maquinas herramientas DUV - 248 Stepper 


\section{Literatura citada}

Adner, R. and Rahul, K. 2010. Value Creation in Innovation Ecosystems: How the Structure of Technological Interdependence Affects Firm Performance In New Technology Generations. Strategic Management Journal, 31: 306-333.

Adner, R. and Kapoor, R. 2016. Innovation Ecosystems and the Pace of Substitution: Re-examining Technology S-curves. Strategic Management Journal, 37: 625648.

Brunner, T. 2003. Why Optical Lithography will live forever.Journal American Vacuum Society. B 21 (6).

Balcer,Y. and Lippman, S. 1984.Technological expectations and adoption of improved technology. Journal of Economic Theory, 34 (2): 292-318.

Broad, W. 1986. La Verdadera Guerra de las Galaxias. Editorial Planeta. Barcelona.

Farzin, Y. and Huisman, K. and Kort, M. 1998. Optimal Timing of Technology adoption. Journal of Economic Dynamics and Control, 22 (5): 779-799.

Funk, J. 2009. Improvements in Components and Discontinuities in Systems: the case of Computers. Summer Conference 2009 in Denmark.

Henderson, R. 1995. Of life cycles real and imaginary: The unexpectedly long old age of optical lithography. Research Policy, (24): 631-643.

Henderson, R. and Clark, K. 1990. Architectural innovation: the reconfiguration of existing product technologies and failure of established. Administrative Science Quaternary, 35 (1):3-90.

Hutcheson, G. 2108. Moore's law, lithography, and how optics drive the semiconductor industry, Proc. SPIE 10583, Extreme Ultraviolet (EUV)Lithography IX, 1058303 (19 March 2018); doi: 10.1117/12.2308299.

Kim, J. 2014. Innovation in the Semiconductor Equipment Industry (Incremental Innovation vs. Radical Innovation). Mg.Sc. Dissertation, The University of Manchester.

Llaque, Luis.2017.Innovación en Tecnologías de Máquinas Herramientas y Modularidad: El caso de la Industria Litográfica en Semiconductores 1985-2010. Universidad Agraria La Molina.Perú.Mimeo.

Marcher, J. and Mowery, D.. 2004. Specialization Vertical and Industry Structure in High Technology industries. Strategic Management, 21: 317-356.

Murmann,,J. and Frenken, K. 2006. Towards a Systematic Framework for Research on Dominant Designs, Technological Innovations and Industrial Change. Research Policy, 35 (7): 925-952.

Marcher, J. 2006. Technological development and the boundaries of the firm: A knowledge - based examination in semiconductor manufacturing. Management Science. 52(6): 826- 843.

Nikon, P. 2005.Next-generation lithography. Dispoible en: http:// www.nikonprecision.com/tech/tech 4 1.htm, accesado en Octubre 2015.

Pieczulewski, C. 1995. Benchmarking Semiconductor Lithography Equipment Development \&Sourcing Practices Among Leading- Edge US. Manufacturs.MIT. Thesis.

Pillai,U. 2013. A model of Technological progress in the microprocessor industry.Journal of Industrial Economics, 61 (5): 877-912.

Pillai,U. 2017.The Emergence of Tools Suppliers in the Semiconductor Industry.Http. Disponible en: www. paper.ssrn.com.

Rosenberg, N. 1979. Tecnología y Economía. Editorial Gustavo Gili, S.A. Barcelona.

Schwab, K. 2017. La cuarta revolución industrial. Editorial Debate. España.

Tushman, M.; Anderson, P. 1990. Technological Discontinuities and Dominant Designs: A Cyclical Model of Technological Change. Administrative Science Quarterly, 35:604-633.

Tushman, M. and Rosenkopf, L., 1992. Organizational determinants of technological change: toward a sociology of technological evolution. Research in Organizational Behavior, 14: 311-347

VLSI Research, "Microlithography and Mask Making", Technical Report, VLSI Research 1992. Available at https://www.chiphistory.org/documents/ microlithography\&mask_making.pdf.

Anexo 1. Costo de las generaciones máquinas herramientas para litografía en semiconductores

\begin{tabular}{lcl}
\hline \multicolumn{1}{c}{ Generación } & $\begin{array}{c}\text { Costo/máquina } \\
\text { Herramienta } \\
\text { (en miles de dólares) }\end{array}$ & $\begin{array}{c}\text { Tecnología de la Máquina } \\
\text { herramienta }\end{array}$ \\
\hline Primera (1962) & 15 & Impresión por contacto \\
Segunda (1972) & 25 & Impresión por proximidad \\
Tercera (1973) & 45 & Proyección Scanner \\
Cuarta (1976) & 60 & E- Beam Writer \\
Quinta (1978) & n.d & X- ray Printer \\
Sexta (1978) & 450 & G- line Stepper \\
Séptima (1985) & 2,300 a 3,000 & I - line Stepper \\
Octava (1986) & 3,600 a 4,200 & DUV- 248 Stepper \\
Novena (1996) & 10,000 a 15,000 & DUV - 193 Stepper \\
Decima (2005) & 36,000 a 50,000 & DUV - 193 Immersion \\
\hline
\end{tabular}

Fuente: Estimaciones sobre la base de Henderson (1995); Pieczulewski (1995);VLS(1992);Adner and Kapoor (2010); Pillai (2013);Hutcheson, G. Dan (2018). 